\title{
Paradis perdu et champs verdoyants : figures de l'utopie dans la pensée et la poésie de Léopold Sédar Senghor
}

\section{Introduction}

« En politique, on est rarement prophète » (1964, p. 8), écrit Léopold Sédar Senghor dans son introduction à la série Liberté qui rassemble ses textes en prose. Nous sommes en 1963, et cela fait trois ans qu'il est le président de la République du Sénégal. Né en 1906, il a connu l'apogée du monde des empires, apogée que l’on peut, dans le cas de la France, identifier à l'Exposition coloniale de 1931, où prenait figure cette utopie qu'était la « Plus Grande France » (voir Wilder, 2005 ; Hodeir et Pierre, 2011). Il faut le rappeler, le drapeau français flottait alors sur tous les continents et, bien qu'en majorité non-citoyens, 110 millions de personnes étaient de nationalité française à la veille de la Seconde Guerre mondiale. Seul un prophète, accomplissant « le tour du monde en un jour ", pour reprendre un slogan publicitaire de l'Exposition, aurait pu prédire qu'à l'écriture de cette introduction, en Afrique, seules la Côte française des Somalis, l'île de la Réunion et les Comores feraient encore partie de la République française. Lui qui était aux premières loges de la décolonisation, mais aussi acteur dans les assemblées où se prirent les décisions qui transformèrent le monde des empires en celui des États souverains indépendants, est donc bien placé pour conclure : «L'essentiel est d'être humble devant les faits : devant la vie» (1964, p. 8).

Plus de soixante-dix ans après l'introduction du principe du « droit des peuples à disposer d'eux-mêmes » dans la charte des Nations unies, il nous est difficile de penser que les acteurs historiques n'étaient pas en mesure de connaitre l'aboutissement

\footnotetext{
- Sébastien Heiniger - postdoctorant à l'Institut des mondes africains de Paris ; e-mail : sebheiniger@gmail.com

ORCID iD : https://orcid.org/0000-0002-6064-2094
} 
du processus de décolonisation. Aujourd'hui, les indépendances semblent naturelles et on se souvient surtout de Senghor comme le théoricien de la Négritude, le " père fondateur " de la Francophonie et le poète élu à l'Académie française en 1983. Ce que l'on oublie, c'est son projet politique et ce qu'il voulait bâtir sur la fondation de la Négritude, comme si cette « humilité » avait effacé le député français. Or un projet politique présuppose aussi une vision de l'avenir, ou, pour prendre une autre métaphore, une utopie à atteindre. Ce sont ces lieux qu'il s'agira de retrouver dans ses essais et poèmes, lieux irréels parce qu'ils ne sont plus ou ne sont pas encore, mais qui n’en demeurent pas moins des figures de l'eutopia qu'il prophétise.

\section{La fonction de l'utopie}

Notre parcours commence par le poème Désespoir du volontaire libre que Senghor écrit avant la débâcle de 1940 et publie en 1948 dans le recueil Hosties noires. Le poète, devenu narrateur dans ce poème, raconte une anecdote dont le protagoniste est un jeune homme africain fraîchement engagé. Ce dernier était prêt à mourir pour la France, mais on lui a donné ce signe de la démarcation sociale qu'est l'uniforme du tirailleur sénégalais. Saisi d’angoisse, il vient de se rendre compte que la ségrégation coloniale se poursuit jusque dans l'armée, ce qu'il considère comme un «nouvel affront » (Senghor, 1990, p. 70) de la France à son égard.

Par le truchement de son narrateur, Senghor parvient à opposer deux perspectives sur la réalité dans ce poème : celle du poète et celle du jeune homme. Placé sur un point élevé, peut-être le toit de la caserne, ce dernier « se penche» et « regarde » :

Il se penche il regarde la cour béante et quatre rangées de fenêtres sous lui

Il se penche, et la plaine apocalyptique est labourée de tranchées, où pourrissent les morts comme des semences infécondes

Il se penche sur de hauts tumulus de solitude.

Et au-delà, la plaine soudanaise que dessèchent le Vent d'Est et les maitres nordiques du Temps

Et les belles routes noires luisantes que bordent les sables, rien que les sables les impôts les corvées les chicottes

Et la seule rosée des crachats pour leurs soifs inextinguibles au souvenir des verts pâturages atlantidiens. (Senghor, 1990, p. 70)

Sous les yeux du jeune homme, la "plaine apocalyptique » - la Grande Guerre devient un champ de culture. Dans cette métaphore que le poète file, les tranchées sont des sillons et les morts des semences. Mais ces semences pourrissent dans la terre. Les poèmes du recueil Hosties noires portent hommage aux tirailleurs sénégalais morts pour la France et ici, comme dans plusieurs autres poèmes, Senghor rappelle que les vies versées en sacrifice n'ont pas porté leurs fruits. Effectivement, 
si certains anciens combattants purent accéder à la citoyenneté française promise ${ }^{1}$, le régime de l'indigénat s'est raidi pendant l'entre-deux-guerres. C'est cette situation coloniale que le jeune homme voit lorsqu'il se penche sur "la plaine soudanaise". Couverte de sable, elle n'est plus que l'ombre des "verts pâturages atlantidiens " ${ }^{2}$ qu'elle était. Le jeune homme se souvient alors des offenses auxquelles s'ajoute le " nouvel affront ": " les impôts, les corvées, les chicottes " sont diverses facettes du régime de l'indigénat ${ }^{3}$ et « la seule rosée des crachats » figure le quotidien empli d'humiliations. Le constat du jeune homme est donc amer, d'autant plus qu'il voit la nouvelle guerre qui se profile à l'horizon :

Il se penche sur une seconde plaine saturée de chéchias et de sang, sur une seconde plaine altérée d'amour comme d'une pluie amicale

Et c'est, jusquà la fusion parallèle, la si fatale succession des plaines et des plaintes silencieusement.

Sur la pointe des pieds, il se penche, se soulève pour percer son désespoir l'horizon. (Senghor, 1990, p. 71)

Cette "seconde plaine " est la Seconde Guerre. Semblable aux deux premières plaines, elle s'annonce "saturée de chéchias et de sang " et "altérée d'amour ". Le jeune homme se soulève et porte son regard vers l'horizon dans une ultime quête de sens, mais il ne voit que «la si fatale succession des plaines et des plaintes", si bien que l'horizon et le désespoir deviennent un. En déséquilibre sur son point de vue élevé, attiré par « l'espace vide et ce vaste pays vidé d'espoir » (Senghor, 1990, p. 71), il est saisi de vertige, chute et trouve la mort.

Le deuxième point de vue est celui du poète-narrateur, qui qualifie le jeune de «naif » (Senghor, 1990, p. 70) au début du poème et de «trop faible enfant » (p. 71) à sa fin. Son commentaire ne porte pas sur la capacité du tirailleur à saisir la réalité dans laquelle il est plongé - il voit le même panorama que le tirailleur -, mais sur son inaptitude à percevoir ce qui se trouve au-delà de l'actualité fatale :

Il ne voit pas que les morts et les terres hautes des morts masquent les champs là-bas qui verdoient dans l'ombre

D’or et d'étoiles constellés, comme arrosés du sang à leurs pieds et des cadavres gras bien nourris.

Peut-il voir le paradis perdu derrière l'horizon des temps fabuleux ? (Senghor, 1990, p. 71)

1. Au sujet de "l'impôt du sang" des tirailleurs sénégalais, voir Michel (2011).

2. Senghor fait ici référence aux travaux de Leo Frobenius, qui était convaincu que la légendaire Atlantis était une civilisation africaine florissante.

3. Au sujet du régime de l'indigénat, voir Merle (2002). 
Invisibles à l'œil désespéré du volontaire libre, deux nouveaux lieux apparaissent dans ces vers. Respectivement "dans l'ombre » et " derrière l'horizon ", ils peuvent être discernés par ceux qui, comme le poète, parviennent à " percer " l'actualité pour voir ce qui se trouve au-delà d'elle. Ainsi ce dernier voit des " champs làbas » sur lequel le jour ne s'est pas encore levé et un " paradis perdu derrière l'horizon ", paradis qui est certes perdu, mais qui est tout autant présent et perceptible que les champs verdoyants. Remarquons que le temps est saisi par une métaphore spatiale dans ces vers et non par une ligne chronologique. Le passé, l'actuel et le futur en deviennent des lieux distincts et coprésents dans l'espace qui se déploie autour du poète. Néanmoins, parce qu'ils sont hors de la réalité circonscrite par l'horizon, ni le paradis perdu ni les champs verdoyants ne sont actuels, et nous pouvons donc les concevoir comme des utopies.

Les utopies forment certes un genre littéraire spécifique et on ne pourrait y classer les textes de Senghor. Son dessein n’a jamais été de créer une "utopie littéraire ", mais de définir un projet politique. Cependant, comme le remarque Paul Ricœur dans L'idéologie et l'utopie, il est difficile de subsumer les utopies sous une définition générale, ce qui nous laisse au moins l'analogie avec l'utopie à disposition pour décrire les lieux irréels du penseur et poète de la Négritude. Pour Ricœur, l'utopie est une pratique imaginative et il l'aborde donc par le biais de ses travaux portant sur la fiction et plus généralement dans le cadre de sa réflexion au sujet du problème philosophique de l'imagination. Son propos est de montrer que les pratiques imaginatives ne sont pas nécessairement des fuites hors du réel, parce que la fiction est dotée d'un pouvoir heuristique qui révèle des possibles et permet d'anticiper l'agir. En tant que " nulle part ", écrit Ricœur, l'utopie nous offre une "place vide d'où nous pouvons réfléchir à nous-mêmes » (1997, p. 35). Grâce à l'utopie, « [1] e champs des possibles s'ouvre largement au-delà de l'existant et permet d'envisager des manières de vivre radicalement autres » (1997, p. 36). Telle est la fonction de base de l'utopie selon le philosophe, celle de jeter une nouvelle lumière sur notre réalité et d'ouvrir le champ de notre imagination aux variations du possible.

Toutefois, quand elle ouvre la voie vers ce qui est au-delà de la réalité, la pratique imaginative peut tant nous perdre dans la fantasmagorie que nous mener dans le possible. Ricœur parle donc de la « double valence» $(1997$, p. 16) de la fiction, laquelle est aussi présente dans la création d'utopies, comme l'indique le jeu entre utopia et eutopia de Thomas More. D’un côté, cette pratique imaginative peut nous faire fuir dans le «non-lieu », de l'autre elle peut nous faire découvrir le «bon lieu ». Il n'en reste pas moins que l'utopie est nécessaire pour Ricœur, parce qu'elle empêche l'" horizon d'attente de fusionner avec le champ de l'expérience » (1997, p. 16). Or, c'est précisément l'incapacité à " percer son désespoir l'horizon » qui mène le jeune homme à subir le vertige du non-sens. Les utopies que sont le paradis perdu et les champs verdoyants redonnent sens et direction, et, ultimement, comme elles demandent à être actualisées, elles peuvent être motrices de la transformation de la réalité. 


\section{Discerner le possible pour changer le réel}

Ricœur trouve matière à réflexion dans Idéologie et utopie de Karl Mannheim et le critère de non-congruence avec la réalité qu'il a proposé pour définir l'utopie (1997, p. 358). C'est sur la base de cette non-congruence avec la réalité que nous proposons de comprendre les lieux irréels qui prennent corps dans les textes et poèmes de Senghor comme des utopies. Selon notre interprétation, par le «paradis perdu derrière l'horizon » ce dernier désigne la " civilisation négro-africaine » qu'il décrit dans ses textes en prose, civilisation disparue depuis sa mise en contact avec la civilisation islamique et l'Europe. Toujours selon notre lecture, les « champs là-bas qui verdoient dans l'ombre » sont la France à venir pour la réalisation de laquelle Senghor militera à l'Assemblée nationale. Ces deux lieux irréels font l'objet des deux parties qui suivent.

\subsection{Le "royaume Nègre type "}

Nous trouvons des descriptions d'États précoloniaux africains dans plusieurs de ses textes ${ }^{4}$. S'il dénonce la «thèse de l'anarchie nègre » et la faible « culture ethnologique et historique " (1964, p. 45) de ceux qui la défendent, nous verrons que son dessein n'est pas uniquement de combler l'ignorance de ses lecteurs et combattre les stéréotypes coloniaux. Dans Ce que l'homme apporte (1939) et Vues sur l'Afrique noire, ou assimiler non être assimilé (1945) qu'il écrit avant de devenir député, Senghor décrit le Royaume du Sine, lequel serait une réalisation du "royaume nègre type » (1964, p. 32). Ce royaume-type est un modèle idéal et n'est donc pas une entité réelle. Or, les États dont il aurait déterminé la forme n'ont pas plus de réalité effective. Le Royaume du Sine, par exemple, a disparu dans les années 1920, quand la royauté a été abolie, pour donner les pleins pouvoirs à l'administration coloniale (1964, p. 57).

Dans l'essai de 1939, Senghor veut présenter " ce que l'homme noir apporte " à l'humanité. Dans son introduction, il écrit que le « Nègre » est « déjà présent dans l'élaboration du monde nouveau » (1964, p. 22). Ce dernier révèle en effet sa "présence actuelle » dans les arts. Ses " présences virtuelles », en revanche, ne sont pas encore réalisées, mais peuvent être entrevues par "l’étude du Nègre " (1964, p. 22). Ces dernières sont les " apports nègres dans le domaine social et politique ", lesquels serviront à «l'élaboration d'un monde plus humain » (1964, p. 33). Il n'est donc peutêtre pas fortuit que la description que Senghor donne du " royaume nègre type " comporte les thèmes utopistes identifiés par Ricœur, dont les questions comme « la société, le pouvoir, le gouvernement, la famille, la religion» (1997, p. 36). Dans

4. Notamment : Ce que l'homme noir apporte (1939); Vues sur l'Afrique noire, ou assimiler, non être assimilés (1945) ; L'Afrique noire, la civilisation négro-africaine (1947); la conférence qu'il a donnée au ${ }^{\mathrm{er}}$ Congrès des Artistes et Écrivains noirs : Lesprit de la civilisation ou les lois de la culture négro-africaine (1956) ; celle donnée au II Congrès des Artistes et Écrivains noirs : Éléments constitutifs d'une civilisation d'inspiration négro-africaine (1959). 
son étude, brossant le portrait de la "société nègre ", Senghor part de la plus petite " cellule sociale » qu'est la "famille négro-africaine », pour atteindre une entité de plus large échelle, laquelle peut être " une confédération ou un empire ", ceci dans le but de montrer que, de la plus petite à la plus large entité, la " communauté » n'est jamais scindée (1964, p. 27-29). Poursuivant son propos, il aborde la question politique et énonce une critique des démocraties occidentales à l'aune d'un système idéal, où gouverner est " tendre au bien des communautés et des personnes : de la Cité " (1964, p. 31). Cet idéal, le royaume du Sine le réalisait. Son assemblée législative était " composée de hauts dignitaires et de notables, chefs de famille claniques ", dont « la sagesse [...] vient de la connaissance de la tradition, de l'expérience de la vie et du sentiment de ses responsabilités " (1964, p. 32). Le pouvoir exécutif était exercé par un roi dont l'autorité n'était pas coercitive, parce qu'il était « un ascendant d'ordre spirituel » honoré par le peuple qui l'élisait «par l'intermédiaire des principaux chefs de famille» $(1964$, p. 32). On le voit ici, cette « communauté harmonieuse » qui est " une cité fondée à l'image des communautés naturelles et reposant sur elles » (1964, p. 32) est aussi l'eutopia de Senghor.

Mannheim classe le conservatisme qui " projette pour l'avenir de restaurer le passé » (Ricœur, 1997, p. 362) parmi les utopies. Senghor défie-t-il l'idéologie coloniale avec une utopie conservatrice ? Certes, il enjoint le politique de revenir aux " sources " (1964, p. 31) du législatif et de l'exécutif, dans Ce que l'homme noir apporte et annonce, dans Vues sur l'Afrique noire, que l'Afrique noire peut aider la France à " redécouvrir son visage ancien, qui est l'authentique, sous les déformations que lui a fait subir son évolution moderne " (1964, p. 69), mais le classer parmi les conservateurs serait faire l'impasse sur l'État dans lequel le passé serait restauré. En effet, dans l'essai de 1939, il écrit que "la solution que le Nègre a donnée aux problèmes sociaux et politiques [...] a répondu, par avance, à cette "unité pluraliste" qui reste l'idéal des humanistes d'aujourd'hui » (1964, p. 29). Ces guillemets signalent expressément sa référence à la pensée fédéraliste de l'entre-deux-guerres, ce qui revient à affirmer que les États de l'Afrique précoloniale réalisaient déjà ce que les fédéralistes ${ }^{5}$ envisageaient pour la France. Il importe toutefois de bien l'entendre, car dans ses textes, le terme « France » désigne l'ensemble de la République impériale. L' ' unité pluraliste » à venir serait à son échelle et, par conséquent, ni la métropole ni le Royaume du Sine ne retrouveraient leurs formes anciennes. Pour le dire en un mot, les champs verdoyants ne sont pas le paradis perdu.

Senghor ne propose pas de restaurer ce qui n'est plus, mais d'en reprendre les principes d'élaboration. En voulant jeter une lumière nouvelle sur ces États précoloniaux grâce à la notion d'utopie, notre propos n'est pas de dire qu'ils sont le fruit de son imagination, mais de comprendre leur fonction dans sa pensée. Comme

5. Il cite notamment Daniel-Rops, Denis de Rougemont et Jacques Maritain, qui sont membres de ce que l'on désigne sous le nom de «non-conformistes des années 30 » et militent parmi les mouvements fédéralistes européens. 
le relève Ricœur, pour Mannheim, l'utopie est fondamentalement réalisable, voire toujours en voie de réalisation $(1997$, p. 359). Son irréalité n’en fait donc pas une fantasmagorie, mais un défi posé à l'ordre établi. Ces États exercent cette même fonction utopique dans la pensée de Senghor. Décrivant d'autres organisations sociétales que la "Civilisation » à laquelle les colonisés devaient progressivement se conformer, ces utopies sapent les justifications et certitudes coloniales. Plus encore, quand il propose de s'inspirer des États précoloniaux négro-africains pour élaborer le monde plus humain, il ne fait pas que décrire ce qui n'est plus, il présente des possibles politiques, dont la France qui vient. Cette utopie est aussi présente dans les poèmes d'Hosties noires.

\subsection{La France qui vient}

Senghor écrit Prière des tirailleurs sénégalais à Paris en avril 1940. Le poète, qui se présente comme intercesseur pour des tirailleurs fraîchement débarqués en Europe, expose leur requête principale dans la quatrième partie du poème :

Seigneur, écoute l'offrande de notre foi militante

Reçois l'offrande de nos corps, l'élection de tous ces corps ténébreusement parfaits

Les victimes noires paratonnerres.

Nous T'offrons nos corps avec ceux des paysans de France, nos camarades

Jusque dans la mort après la première poignée de main et les premières paroles échangées

$[\ldots]$

Que l'enfant blanc et l'enfant noir - c'est l'ordre alphabétique -, que les enfants de la France Confédérée aillent main dans la main

Tels que les prévoit le Poète, tel le couple Demba-Dupont sur les monuments aux Morts

Que l'ivraie de la haine n’embarrasse pas leurs pas dépétrifiés. (Senghor, 1990, p. 74)

Trois générations de représentants de peuples se donnent la main dans cette strophe : " le couple Demba-Dupont ", morts à la Grande Guerre ; les "victimes noires » et les « paysans de France » qui vont mourir au front; l'enfant blanc et l'enfant noir de la France Confédérée. Inaugurée le 30 décembre 1923 au rond-point de l'Étoile à Dakar, la statue de bronze «Demba et Dupont » représente le lien de fraternité né dans les tranchées de la Grande Guerre ${ }^{6}$. Le Poète, qui annonce que les pas des enfants seront " dépétrifiés ", voit s'incarner dans la génération naissante la promesse figurée dans cette statue. La sculpture est ainsi commémorative et annonciatrice, mais pour que la France Confédérée advienne, il faut que les frères issus de peuples différents soient unis par des liens de fraternité qui prolonge celle des armes, c'est-àdire par la fraternité française.

6. Pour une histoire de ce monument, voir Michel (2001). 
Trois mois après la débâcle de juin 1940, Senghor écrit Au Guélowâr au Camp d'Amiens. Dans les derniers vers de ce poème, les tirailleurs devenus prisonniers annoncent au général de Gaulle qu'ils resteront fidèles à la France :

Ta voix nous dit la République, que nous dresserons la Cité dans le jour bleu

Dans l’égalité des peuples fraternels. Et nous nous répondons : «Présents, ô Guélowâr ! » (Senghor, 1997, p. 77)

Cette France qui vient est décrite comme une Cité unissant une pluralité de peuples fraternels et égaux. Sans entrer dans les détails, il est à relever qu'une telle utopie demande de redéfinir un grand nombre des principes constitutifs de la République, où l'égalité concerne des individus qui forment un peuple unique.

On le voit, présentant la France Confédérée et la Cité dans ces poèmes, Senghor n'en fait pas des lieux inaccessibles. Bien qu'inactuelle, cette République utopique existe en puissance et n'attend que son édification. Senghor n'est cependant pas naïf quant aux conditions de réalisation d'un tel État. Ainsi, dans le premier poème, il enchâsse la prévision du Poète dans une prière. Il est certes un catholique consistant et cette prière est adressée au Seigneur, mais l'intervention attendue est humaine et non divine, car ce poème est destiné à des lecteurs. C'est eux et non le Seigneur que ces vers doivent mouvoir. Similairement, dans le deuxième poème, les tirailleurs répondent "présents » tant à l'appel du général, qu’à l'œuvre d'édification de la Cité à venir. S'ils adhèrent à la France libre, le «Et » du dernier vers instaure aussi une condition, laquelle est que de Gaulle tienne sa promesse. En somme, entre l'actualité et l'utopie, une voie se dessine, celle de la réforme de la France, plus précisément, celle de la décolonisation par fédéralisation de la République pour atteindre l'eutopia où les peuples sont égaux et fraternels. Il n'en demeure pas moins que son avènement est le fruit de choix.

\section{4. « Gouverner, c'est prévoir »}

Dire qu' « en politique, on est rarement prophète » n'est pas dire qu'on ne l'est jamais. Avant que Senghor intègre à sa pensée le terme "prospective ", au début des années 1960 (concept qu'il reprend du philosophe Gaston Berger), son verbe clé est " prévoir », qui, ci-dessus, est l'activité du Poète. On retrouve aussi ce verbe dans Pour une solution fédéraliste qui est l'étude sur la révision du titre VIII de la Constitution qu'il remet en 1955 à Edgar Faure, lequel est alors le président du Conseil des ministres de la IVe République. Dans la conclusion de son rapport, Senghor rappelle que " gouverner, c'est prévoir» (1971, p. 168) et ajoute qu' "il est facile de prévoir, dans un monde en mouvement que, dans dix ans, il n'y aura plus de "peuple dépendant" " (1971, p. 168). 
Nous pouvons alors reformuler de la sorte la phrase de son introduction à Liberté II : " en politique, on se doit de prévoir, mais on est rarement prophète ». La Négritude émerge certes avant la guerre, mais Senghor en développe la théorie quand il est député à l'Assemblée nationale de la $\mathrm{IV}^{\mathrm{e}}$ République, c'est-à-dire dans un contexte où la fin de la dépendance coloniale n'est pas une éventualité. Il écrit son rapport en pleine guerre froide et tant les États-Unis que l'URSS cherchent à démanteler les Empires européens. En outre, vingt-neuf nations nouvellement indépendantes viennent de se réunir à Bandoeng, où elles ont condamné le principe même de la colonisation. Inéluctable, la décolonisation de l'Afrique française n’était pas pour autant prédéfinie et comme Senghor lécrit à Faure en conclusion de son rapport « La France se doit donc de prévoir [...] et de choisir » (1971, p. 168). Un an plus tard, avec l'adoption de la loi-cadre Defferre, le choix était fait et le cours des événements s'est définitivement tourné vers les indépendances de 1960.

L'utopie de Senghor ne s'est pas réalisée, mais était-il un penseur utopique ? Comme l'écrit Ricœur, "Il [le concept d'utopie] est considéré comme représentant une espèce de rêve social qui ne se soucie guère des étapes réelles nécessaires à la construction d'une nouvelle société » (1997, p. 17-18). Transformer la République impériale en République fédérale relevait certes de l'utopie, mais certaines victoires, comme la promulgation du Code du travail de 1952, allaient vers sa réalisation. La part de rêve était certes présente, mais le pari de l'éthique l'était tout autant, le pari d'établir l'égalité de droits politiques, civiques et sociaux entre membres d'un État plurinational.

\section{Conclusion}

Aujourd'hui, dans l'Afrique francophone et sa diaspora, la mentalité utopique semble trouver un deuxième souffle, après les coups que lui a asséné l'afropessimisme. En 2016, Felwine Sarr et Achille Mbembe ont convié les intellectuels et artistes aux Ateliers de la pensée, qui sont depuis devenus le rendez-vous intellectuel le plus important en Afrique francophone. La même année, Sarr publiait son essai Afrotopia, lequel a connu un succès certain. "Fonder une utopie ", écrit-il, " ce n'est point se laisser aller à une douce rêverie, mais penser des espaces du réel à faire advenir par la pensée et l'action " (2016, p. 14). Sous sa plume, l'Afrique redevient un lieu riche de potentialités qui attend " une pensée qui porte sur le destin du continent africain » (2016, p. 14).

Comme l'écrit Senghor, l'Afrique a vécu " les deux cents millions de morts de la Traite des Nègres, les violences de la Conquête, les humiliations de l'Indigénat » (1964, p. 9). Aujourd'hui, bien des termes pourraient être ajoutés à cette liste. Chez Sarr, comme chez Senghor, cette réalité n'est pas niée, mais pour tous les deux, il importe de percevoir l'Afrique qui vient et d'orienter nos choix en vue de sa réalisation. 
« Le concept d'utopie est un outil polémique : il appartient au champ de la rhétorique ", écrit Ricœur (1997, p. 406). L’enjeu reste en effet de persuader le lecteur. Pendant quelques instants, ce dernier admet les prémisses de la fiction et, avant de revenir à celles de la réalité, il aura peut-être prévu ce qui adviendra. Ses attentes à l'égard du futur transformées, il sera peut-être prêt à faire le pari de l'éthique et agir pour la réalisation d'un " projet de civilisation qui met l'homme au cœur de ses préoccupations» (Sarr, 2016, p. 15). Ce sera à l'avenir de nous dire si les réflexions « sur le présent et les devenirs d'une Afrique au cœur des transformations du monde contemporain " (Mbembe et Sarr, 2017, p. 8) ont dessiné une utopie irréalisable ou entraîné nos pas vers l'eutopia tant rêvée.

\section{RÉFÉRENCES}

Hodeir, C. et Pierre, M. (2011). L’Exposition coloniale, Paris 1931. Paris-Bruxelles: André Versaille. Mbembe, A. et Sarr, F. (dir.). (2017). Écrire l'Afrique-Monde. Paris : Philippe Rey. Dakar : Jimsaan.

Merle, I. (2002). Retour sur le régime de l'indigénat : genèse et contradictions des principes répressifs dans l'empire français. French Politics, Culture \& Society, 20(2), 77-97.

Michel, M. (2001). Hosties noires entre mémoire et reconnaissance. Dans J. Girault et J. Lecherbonnier (dir.), Léopold Sédar Senghor : Africanité-Universalité (p. 102-110). Paris : L'Harmattan.

Michel, M. (2011). « Avant j’etais nègre, maintenant je suis français ». Les soldats noirs de la Grande Guerre. Dans R. Belot (dir.), Tous républicains! Origine et modernité des valeurs républicaines (p. 281-290). Paris: Armand Colin.

Ricœur, P. (1997). Idéologie et utopie. Paris : Seuil.

Sarr, F. (2016). Afrotopia. Paris : Philippe Rey.

Senghor, L. S. (1964). Liberté I : Négritude et humanisme. Paris : Seuil.

Senghor, L. S. (1971). Liberté II : Nation et voie africaine du socialisme. Paris : Seuil.

Senghor, L. S. (1990). Euvre poétique. Paris : Seuil.

Wilder, G. (2005). The French Imperial Nation-State, Négritude and Colonial Humanism between the Two World Wars. Chicago-London : The University of Chicago Press.

RÉSUMÉ : Léopold Sédar Senghor était un penseur et poète de la Négritude, et il était aussi un homme politique, député à l'Assemblée nationale française dans un contexte où la décolonisation était inéluctable. Avec le soutien théorique de Paul Ricœur, cet article explore l'utopie chez Senghor pour réfléchir à la fonction qu'occupent ces lieux irréels dans sa pensée et restaurer la vision de l'avenir qui était la sienne. Figures de son eutopia, tant le Royaume du Sine que la France Confédérée se proposaient comme communautés harmonieuses à l'aune desquelles imaginer l'avenir. Si Senghor ne défie pas l'idéologie coloniale avec une utopie conservatrice, où le Royaume du Sine retrouverait sa forme ancienne, mais bien avec celle d'une France décolonisée parce 
que fédérale, où il existerait une égalité de droits politiques, civiques et sociaux entre membres de cet État plurinational, la question de savoir s'il était utopiste demeure.

Mots-clés: Léopold Sédar Senghor, Paul Ricœur, République fédérale française, décolonisation, Afrotopia

\section{Paradise lost and lush green fields: figures of utopia in the writings of Léopold Sédar Senghor}

ABSTRACT: Léopold Sédar Senghor was a thinker and poet of Négritude, and also a politician, a member of the French National Assembly in the context where decolonization was inevitable. With the theoretical support of Paul Ricœur, this article explores Senghor's utopia in order to reflect on the function of these unreal places in his thought and to restore his vision of the future. Both the Kingdom of the Sine and Confederate France - the figures of his eutopia - were presented as harmonious communities by which to imagine the future. If Senghor does not challenge colonial ideology with a conservative utopia, where the Kingdom of the Sine would regain its ancient form, but with that of a federal thus decolonized France, where equality of political, civic and social rights between members of a plurinational state would obtain, the question of knowing if he was a utopian remains.

Keywords: Léopold Sédar Senghor, Paul Ricœur, French Federal Republic, decolonization, Afrotopia 